\section{A glycerophospholipid-specific pocket in the RVFV class II fusion protein drives target membrane insertion}

\author{
P. Guardado-Calvo, ${ }^{1,2 *}$ K. Atkovska, ${ }^{3}$ S. A. Jeffers, ${ }^{1,2} \dagger$ N. Grau, ${ }^{1,2} \ddagger$ M. Backovic,,${ }^{1,2}$ \\ J. Pérez-Vargas, ${ }^{1,2} \ddagger$ S. M. de Boer, ${ }^{4} \S$ M. A. Tortorici, ${ }^{1,2}$ G. Pehau-Arnaudet, ${ }^{5}$ J. Lepault, ${ }^{6}$ \\ P. England, ${ }^{5,7}$ P. J. Rottier, ${ }^{4}$ B. J. Bosch, ${ }^{4}$ J. S. Hub, ${ }^{3 *}$ F. A. Rey ${ }^{1,2 *}$
}

\begin{abstract}
The Rift Valley fever virus (RVFV) is transmitted by infected mosquitoes, causing severe disease in humans and livestock across Africa. We determined the x-ray structure of the RVFV class II fusion protein Gc in its postfusion form and in complex with a glycerophospholipid (GPL) bound in a conserved cavity next to the fusion loop. Site-directed mutagenesis and molecular dynamics simulations further revealed a built-in motif allowing en bloc insertion of the fusion loop into membranes, making few nonpolar side-chain interactions with the aliphatic moiety and multiple polar interactions with lipid head groups upon membrane restructuring. The GPL head-group recognition pocket is conserved in the fusion proteins of other arthropod-borne viruses, such as Zika and chikungunya viruses, which have recently caused major epidemics worldwide.
\end{abstract}

$\mathrm{T}$ he Rift Valley fever virus (RVFV), a member of the genus Phlebovirus within the order Bunyavirales (https://talk.ictvonline. org/taxonomy/), is a mosquito-borne virus first isolated in Kenya in 1930 (1). RVFV is responsible for the occurrence of epizoonoses throughout Africa, with devastating economic consequences for the livestock industry (2), and can cause serious disease in humans (3). In 2000, a large-scale RVFV outbreak in the Arabian Peninsula showed that the virus can potentially invade other continents (4). The fact that RVFV can be transmitted to humans by several different mosquito species, a number of which are spread across the planet, is a source of considerable concern (5).

RVFV virions display two envelope proteins, Gn and Gc, at their surface; these proteins are associated as heterodimers arranged with icosahedral symmetry $(6-8)$. The virus enters cells

\footnotetext{
${ }^{1}$ Institut Pasteur, Département de Virologie, Unité de Virologie Structurale, 75724 Paris Cedex 15, France. ${ }^{2}$ UMR 3569 Virologie, CNRS-Institut Pasteur, 25-28 Rue du Docteur Roux, 75015 Paris, France. ${ }^{3}$ Institute for Microbiology and Genetics, University of Goettingen, Justus-von-Liebig weg 11, 37077 Göttingen, Germany. ${ }^{4}$ Virology Division, Department of Infectious Diseases and Immunology, Faculty of Veterinary Medicine, Utrecht University, Utrecht, Netherlands. ${ }^{5}$ UMR 3528 , CNRS, Institut Pasteur, 25-28 rue du Docteur Roux, 75015 Paris, France. ${ }^{6}$ Institut de Biologie Intégrative de la Cellule, CNRS (UMR 9198), Gif-sur-Yvette, France. ${ }^{7}$ Proteopole, Plateforme de Biophysique des Macromolécules et de leurs Interactions (PFBMI), Institut Pasteur, 25-28 rue du Dr Roux, F-75724 Paris Cedex 15, France.

*Corresponding author. Email: pablo.guardado-calvo@pasteur.fr (P.G.-C.); jhub@gwdg.de (J.S.H.); felix.rey@pasteur.fr (F.A.R.) †Present address: Brammer Bio, Cambridge, MA, USA. ‡Present address: Centre International de Recherche en Infectiologie, International Centre for Infectiology Research, Team Enveloped viruses, vectors and immunotherapy, INSERM U1111, Université de Lyon, Lyon, France. §Present address: Department of Viroscience, Erasmus Medical Center, Rotterdam, Netherlands.
}

via receptor-mediated endocytosis (9), with the glycoprotein Gc catalyzing an acid-induced membrane fusion reaction with the endosome for entry. The x-ray structure of RVFV Gc in its prefusion form (10) shows the characteristic fold of class II membrane fusion proteins, initially identified in other arboviruses such as those in the genus Flavivirus (11) (which includes Zika, dengue, and yellow fever viruses) and in the genus Alphavirus (12) (which includes the pathogenic chikungunya virus). Class II membrane fusion proteins encompass homologous proteins with a characteristic three-domain $\beta$-sheet-rich fold, lacking the typical central $\alpha$-helical coiled coil of class I and III fusion proteins (13). Despite their unrelated molecular architectures, all three classes appear to use the same overall fusogenic mechanism, with an initial conformational change allowing exposure of a nonpolar segment-termed "fusion loop" or "fusion peptide"-that inserts into the target membrane while the protein adopts an extended intermediate conformation bridging the two membranes. This intermediate then collapses into a "hairpin" that brings the membranes into close apposition (14). The initial insertion into the target membrane is a crucial step and is best understood for the class I fusion proteins, where it involves a structural reorganization of the fusion peptide to expose a highly hydrophobic platform projecting multiple bulky nonpolar side chains (9 to 20, depending on the virus) inserting into the outer lipid leaflet $(15,16)$. Efficient insertion also requires specific lipids (17). In contrast, the class II and III fusion loops are integral parts of the folded protein and undergo no major reorganization during the fusogenic conformational change $(18-22)$. The energetics of insertion are not understood, as the fusion loops expose only two bulky nonpolar side chains for insertion into the membrane, which is not sufficient to provide strong enough membrane anchoring to withstand downstream forces throughout the fusion process. A requirement for specific lipids has also been documented for class II proteins (23-25), and a direct interaction with cholesterol was demonstrated by photochemical cross-linking in the case of the alphaviruses (26). Furthermore, changes in lipid requirements for insertion correlate with potential alphavirus mosquito vector usage (27), as a variant with a single A226 $\rightarrow$ V226 (A226V) mutation in the fusion protein affects interactions with lipids, which in turn allowed transmission by a different mosquito species to cause a major outbreak of chikungunya disease in Indian Ocean territories in 20052006 (28).

We report here structural, biochemical, and molecular dynamics (MD) studies showing that RVFV Gc has a binding pocket for binding glycerophospholipid (GPL) head groups tucked underneath the fusion loop, resulting in a multitude of polar interactions for binding with high affinity, despite insertion of only two bulky nonpolar side chains per subunit. We also show that additional smallhead-group lipids (in this case, cholesterol) are required to create space between bulky head groups to allow fusion loop insertion. Finally, we show that a similar pocket is present in the flavivirus and the alphavirus class II fusion proteins and that the residue controlling the selection of the bound GPL corresponds to the site of the A226V mutation in chikungunya virus mentioned above.

We crystallized the recombinant ectodomain of RVFV Gc (29) and determined the structure by molecular replacement (table S1), showing a trimer with the typical organization of its three domains in the postfusion form (Fig. 1), as demonstrated recently for a related tick-borne phlebovirus, the severe fever with thrombocytopenia syndrome virus (SFTSV) (20). The new feature provided by the RVFV Gc structure is the C-terminal region downstream domain III (the "stem") (Fig. 1, $\mathrm{A}$ and B, and fig. S1), which was not resolved in the structures of postfusion class II fusion proteins of arboviruses reported to date. This segment adopted an extended conformation, making two additional $\beta$ strands running on the side of the elongated tip of domain II, to reach the fusion loop and complete the Gc postfusion hairpin (Fig. 1B). The observed path of the stem is very similar to that of its counterpart in the somatic cell class II fusion protein EFF-1 from Caenorhabditis elegans (30). Comparison with the prefusion form (10) showed that domains I and II remain as a single rigid body with no hinge in between (fig. S2), a notable difference with the alphavirus $(19)$ and flavivirus $(18,21)$ fusion proteins. All structural changes are confined to the end of domain I opposite to domain II, 
where both domain I $\beta$ sheets are substantially reorganized due to the insertion of an additional $\beta$ strand, $J_{0}$ (fig. S2D and movies S1 to S3), entailing changes in the hydrophobic core of the $\beta$ sandwich.
RVFV Gc crystals grew in a solution containing MES [2-( $N$-morpholino)-ethanesulfonic acid] buffer, and an MES molecule was found bound in a pocket lined by strictly conserved residues adjacent to the fusion loop (fig. S3).
Because MES mimics a zwitterionic lipid head group, we tested cocrystallization in the presence of zwitterionic lipids with short aliphatic tails for increased solubility. These experiments led to crystals of a different space group
Fig. 1. Structure of the postfusion trimer of RVFV Gc and the lipid-head-group binding pocket.

(A) Organization of the RVFV middle (M) genomic segment. Oblique stripes mark the noncoding $3^{\prime}$ and $5^{\prime}$ ends, with the single open-reading frame in between. The signal sequences for the NSm, Gn, and Gc proteins are shown in light blue, and predicted transmembrane regions are in dark gray. The first residue of each mature protein is indicated above. The second row outlines the domain organization of Gc, colored according to domains, with domain I red, the two segments that make up domain II distinguished in yellow and beige, domain III blue, and the stem magenta. Regions not present in the structure are in gray [membrane-proximal region (MPR) and cytosolic tail] or black [transmembrane (TM) segment]. This color scheme is maintained throughout the paper. vRNA, viral RNA. (B) Gc postfusion trimer in surface representation, except for the protomer in the foreground, which is represented as ribbons colored by domains. Disulfide bonds are shown as green sticks and are numbered. The bound $\mathrm{C}_{3} \mathrm{PC}$ lipid (framed) and the $\mathrm{N}$-linked glycan chains are drawn as sticks colored according to atom type (carbon, white; nitrogen, blue; oxygen, red; phosphorus, orange). Lowercase letters denote $\beta$ strands in domain II, following the standard nomenclature for class II fusion proteins. (C) Comparison of the chemical structures of MES and $\mathrm{C}_{3} \mathrm{PC}$. (D) $\mathrm{C}_{3} \mathrm{PC}$ binding groove. The Gc trimer is shown
A
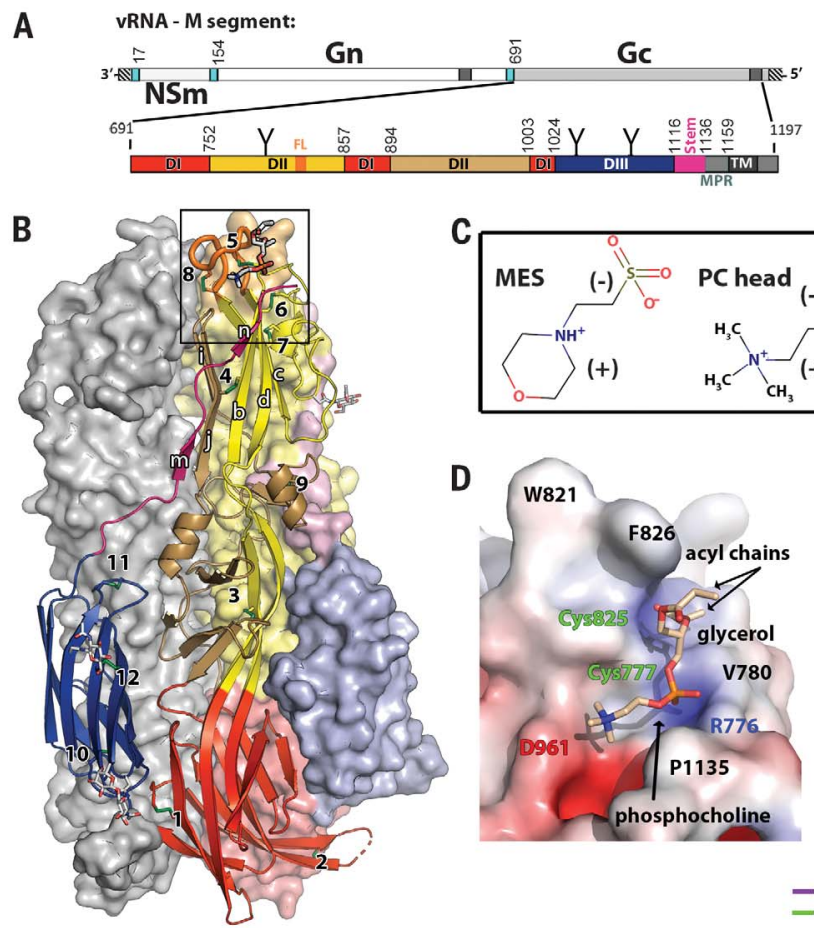

D

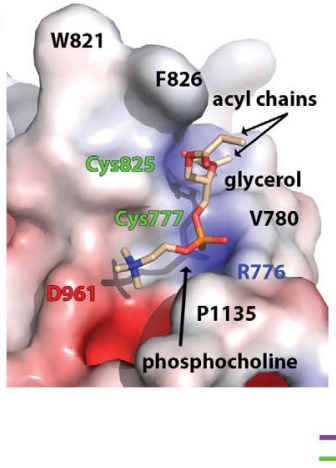

E

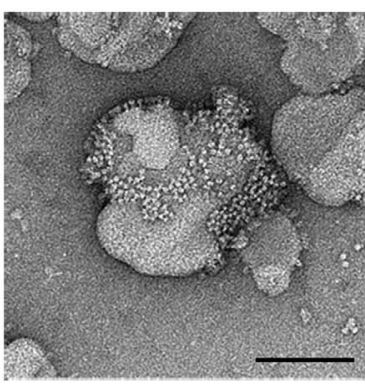

$\mathbf{F}$

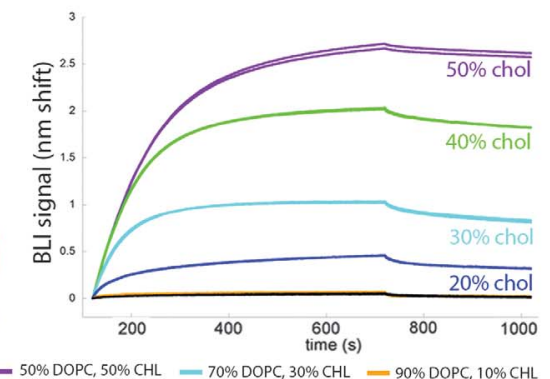

$-50 \% \mathrm{DOPC}, 50 \% \mathrm{CHL}=70 \% \mathrm{DOPC}, 30 \% \mathrm{CHL}=90 \% \mathrm{DOPC}, 10 \% \mathrm{CH}$
$-60 \% \mathrm{DOPC}, 40 \% \mathrm{CHL}-80 \% \mathrm{DOPC}, 20 \% \mathrm{CHL}-100 \% \mathrm{DOPC}$

in surface representation and colored according to electrostatic potential, with $\mathrm{C}_{3} \mathrm{PC}$ shown as sticks. Relevant residues are labeled. (E) Electron micrograph of liposomes after incubation with Gc and negative staining with uranyl acetate. Scale bar, $100 \mathrm{~nm}$. (F) Binding of Gc to DOPC membranes requires cholesterol. Sensorgrams measured by bio-layer interferometry (BLI), showing the interaction of $\mathrm{Gc}$ with liposomes containing different amounts of DOPC and cholesterol (CHL) as indicated.
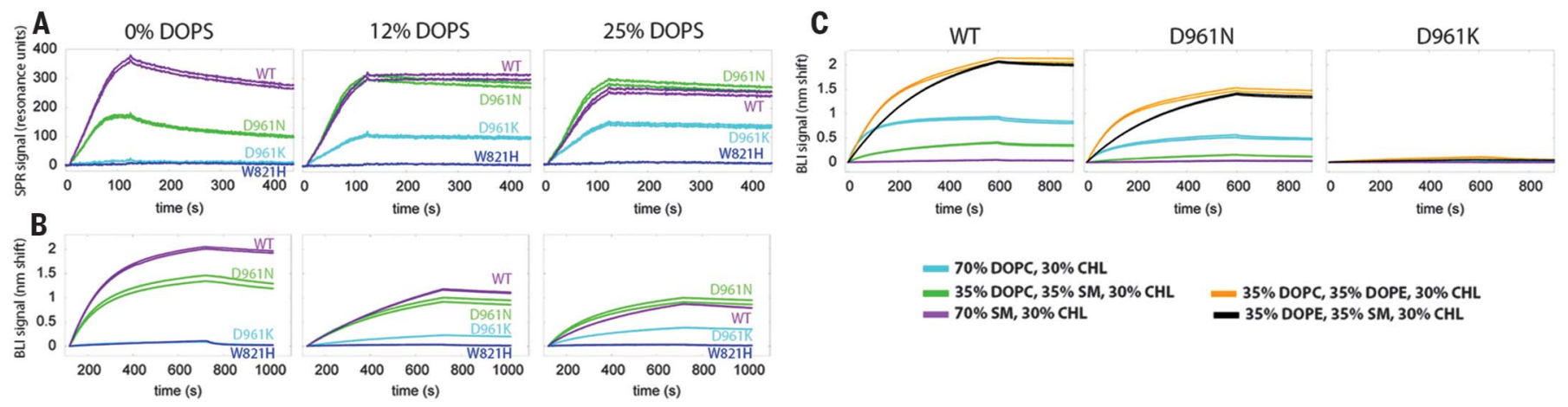

Fig. 2. Lipid binding specificity. Comparative binding of WT Gc and mutants at position 961 (ij loop) to liposomes made of DOPC:DOPS:cholesterol at molar ratios 4:0:4 (left), 3:1:4 (middle), and 2:2:4 (right) (i.e., 50\% cholesterol and increasing DOPS concentration). (A and $\mathbf{B}$ ) Surface plasmon resonance (SPR) (A) and BLI (B) sensorgrams measured with the WT Gc ectodomain (magenta) and with mutants D961N (green), D961K (light blue), and W821H (dark blue, used as control). All experiments were performed as duplicates (shown by

double lines). (C) BLI sensorgrams showing the interaction of Gc WT, D961N, and D961K with liposomes made of 30\% cholesterol and different compositions of SM, DOPC, and DOPE as indicated [note that this color key corresponds only to $(C)]$. In the SPR experiments $(A)$, the liposomes were immobilized on the chip surface and the protein flown on top, whereas in the $\mathrm{BLI}$ experiments [(B) and $(C)]$, the protein was immobilized in the tip and the liposomes were in solution. 
diffracting to higher resolution (table $\mathrm{S} 1$ ) in the presence of the GPL dipropionyl phosphatidylcholine $\left(\mathrm{C}_{3} \mathrm{PC}\right)$. The crystal structure revealed a $\mathrm{C}_{3} \mathrm{PC}$ molecule (Fig. 1D) bound in the same way as MES (fig. S3), with the two nonesterized oxygen atoms of the phosphate group engaging in bidentate hydrogen bonds with the R776 side chain at the very end of the $\beta$ strand $b$ at the tip of domain II (see Fig. $1 \mathrm{~B}$ and fig. S1 for class II secondary structure nomenclature). The glycerol moiety fit between the disulfide bond 5 , which is conserved across all class II fusion proteins, and the side chain of V780 at the end of $\beta$ strand $b$. The two propionyl aliphatic tails projected out, at the very top, packing against the side chain of F826 (Fig. 1D and fig. S3C), the only nonpolar side chain of the fusion loop ( $c d$ loop) that is strictly conserved across the Phlebovirus genus. In line with this observation, the F826N mutation in RVFV Gc abolishes fusion in a cell-to-cell membrane fusion assay (31), and the F699S mutation of its counterpart in SFTSV (fig. S4A) results in nonviable virus (20). The presence of the bound lipid did not alter the conformation of the fusion loop main chain compared to its prefusion form (fig. S4A). Notably, the fusion loop of the flavivirus fusion protein was also in the same conformation in both its pre- and postfusion forms $(18,21)$, unlike the alphavirus fusion protein, which has a longer fusion loop found in alternative conformations $(12,19,32)$.

Liposome coflotation experiments in density gradients, together with electron microscopy, showed that the wild-type Gc ectodomain binds to phosphatidylcholine (PC)-containing liposomes, with the Gc ectodomains bound to membranes via the tapered end (i.e., via the domain II tip) and making clusters at the liposome surface (Fig. 1E and fig. S4B), as shown earlier for other class II fusion proteins $(18,33)$. To further understand the lipid requirements for binding, we monitored the interaction with liposomes containing PC as the main component and varying amounts of other lipids. The Gc ectodomain bound to dioleoyl phosphatidylcholine (DOPC) liposomes at neutral and acidic $\mathrm{pH}$ (fig. S4C) only if the liposomes had at least $20 \%$ cholesterol. Binding increased to be highest at the maximum cholesterol/PC ratio tested (50\%) (Fig. 1F). In the structure, the positively charged
A

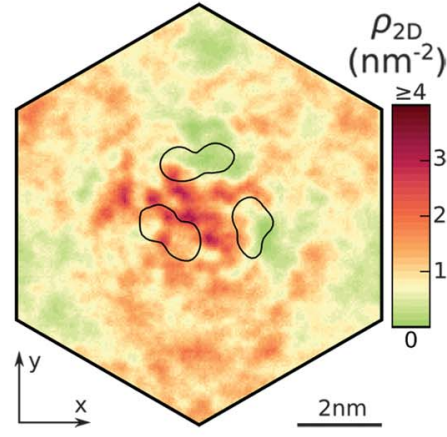

D

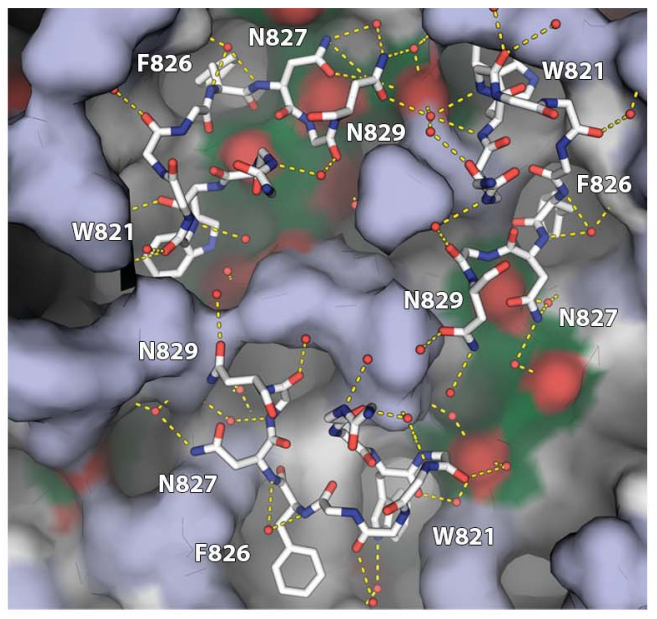

B

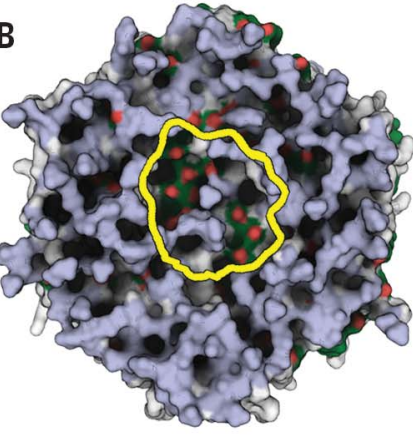

C
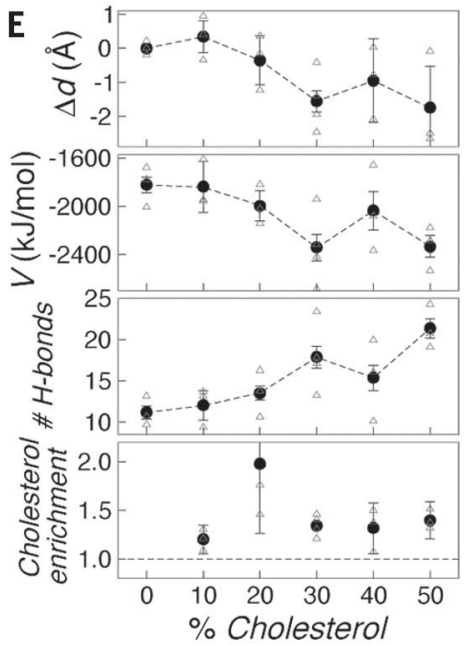

Fig. 3. MD simulations show cholesterol clustering at the protein contact site in the membrane. (A) Cholesterol two-dimensional density $\rho_{2 D}$ in the outer leaflet averaged over a total of $\sim 2 \mu \mathrm{s}$ of simulations at a $40 \%$ cholesterol/PC ratio (table S2 and figs. S6 and S7). The image is color coded according to cholesterol density, as shown in the key at right. (B to D) Simulation snapshots at a $40 \%$ cholesterol/PC ratio. (B) Membrane after removing the protein and solvent. Yellow, protein insertion region; green, cholesterol; red, hydroxyl group; light blue, DOPC head groups; white, DOPC tails. (C) Side view of the MD system after removing the front part of the membrane. Same color code as in (B), with amino acids in the fusion loops shown as thick yellow sticks and DOPCs' aliphatic tails in white or purple (for DOPC inserted into the GPL pocket). (D) Close-up [view corresponding to (B)] of lipid-protein interactions, with the residues of the cd loop represented as sticks. Carbon, nitrogen, and oxygen in white, blue, and red, respectively. Hydrogen bonds are shown as dashed lines. (E) MD simulation statistics as function of DOPC/cholesterol ratio. (Top panel) Membrane penetration depth ( $\Delta d$; see materials and methods) relative to $0 \%$ cholesterol. (Middle panels) Total protein-lipid potential energy $V$ and number of protein-lipid hydrogen bonds, respectively. (Bottom panel) Cholesterol enrichment at the membrane-protein contact area, computed as described in (29). Circles represent average values from $2 \mu \mathrm{s}$ of simulation collected from several independent runs, with error bars (SEM) calculated by block averaging. The average values from individual simulation runs are shown as triangles. For a full description, see (29). 
trimethylamine group of the zwitterionic PC head group faces the strictly conserved D961. Thus, we explored the effect of neutralizing and reversing the electrostatic charge at this location on liposome-binding properties by making mutants D961N and D961K. The WT Gc ectodomain bound consistently better in the absence of dioleoyl phosphatidyl serine (DOPS), a GPL with a negatively charged head group (Fig. 2), whereas the D961N mutant bound to a similar extent with or without DOPS. The D961K mutant had the reverse effect compared with the wild type: The mutant showed almost no binding in the absence of DOPS, and although it remained poor, binding increased with increasing DOPS, as predicted from the positive charge at position 961 in the $i j$ loop. Thus, the residue at the tip of the $i j$ loop affects the membrane-binding properties and is important to specify the GPL being recognized.

We further analyzed lipid specificities by replacing $\mathrm{PC}$ with sphingomyelin (SM), which also has a phosphocholine head group but is connected to a ceramide instead of a diacylglycerol backbone (fig. S5A). The Gc ectodomain did not bind to $100 \%$ SM liposomes, and the addition of cholesterol at various ratios did not rescue binding (fig. S5B). Because liposomes containing SM and cholesterol did not support Gc insertion, we also tried replacing PC with phosphatidylethanolamine (PE), as $\mathrm{PE}$ does not make lipid bilayers by itself and cannot be tested on its own. Adding PE efficiently restored binding (Fig. $2 \mathrm{C}$ and fig. S5C), which led to increased binding with respect to the liposomes containing PC and cholesterol. The effect of the mutants at position 961 was the same as for the PC-containing liposomes: The neutral D961N mutant bound less well, and the D961K mutant did not bind appreciably (fig. S5C).

To obtain a molecular model for Gc-membrane interactions, we ran MD simulations of the Gc trimer in contact with membranes composed of DOPC and cholesterol at different ratios (fig. S6) (29). In agreement with the liposomebinding experiments, the simulations showed that, with increasing cholesterol content, the protein reached deeper into the membrane and formed more favorable contacts (Fig. 3), as quantified by a decrease in protein-lipid potential energies and an increase in the number of protein-lipid hydrogen bonds (Fig. 3E and movie S5). The enhanced protein-lipid interactions correlated with an enrichment of cholesterol at the protein-membrane interface [Fig. 3, A and E (bottom panel), and fig. S7A]. Cholesterol promoted binding mainly by creating space between the bulky DOPC head groups (Fig. 3, B and C), allowing the Gc fusion loop to penetrate the head-group region (Fig. 3D). In addition, multiple hydrogen bonds with cholesterol were formed by the main chain of the fusion loop and with side chains of inserted residues such as W821, N827, and N829 (Fig. 3D), in line with the reported lack of fusion activity of the mutant N827A (31).
In the MD simulations, DOPC head groups consistently found their way into the GPL pocket (which was left empty by removing the bound $\mathrm{C}_{3} \mathrm{PC}$ molecule at the beginning of the calculations), demonstrating that the lipid-binding mode revealed in the crystal structure is also used in a membrane environment. The simulations revealed long-lived strong hydrogen bonds between the DOPCphosphate group and the R776 guanidinium
A

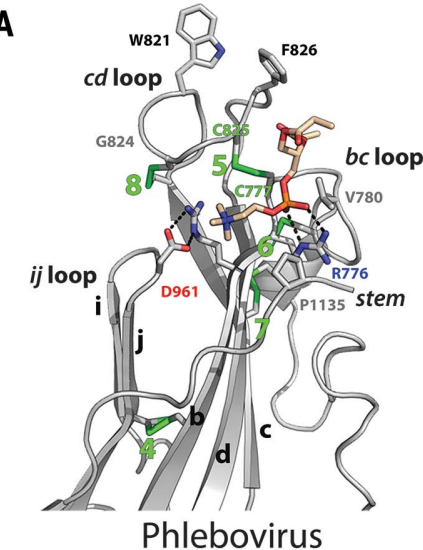

Phlebovirus

b

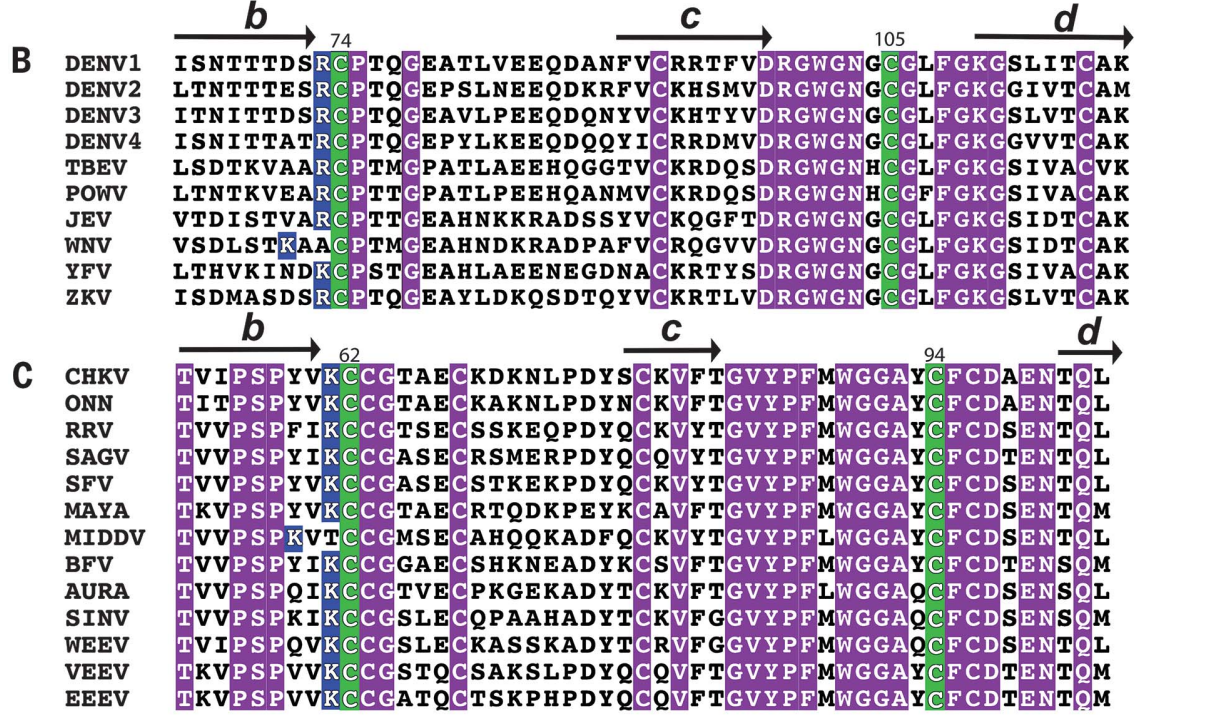

Fig. 4. The GPL binding pocket is conserved in the flavivirus and alphavirus fusion proteins. (A) The left panel shows a cartoon representation of the $\mathrm{C}_{3} \mathrm{PC}$ binding site in RVFV Gc, with important functional residues shown as sticks and labeled. The $\mathrm{C}_{3} \mathrm{PC}$ molecule is shown as sticks colored by atom type, with carbons in light pink. The Flavivirus $\mathrm{E}$ protein from dengue virus serotype 1 (PDB code 4GSX) (middle panel) and the Alphavirus E1 protein from Semliki Forest virus (PDB 1RER) (right panel) are displayed in the same orientation, with a $\mathrm{C}_{3} \mathrm{PC}$ molecule modeled based on the structure of RVFV Gc. The conserved disulfide bond, the first residue of the $b c$ loop, and residues at the tip of the ij loop are represented as sticks and labeled. The mutation affecting the lipid dependence of alphavirus E1 (position 226) is indicated. Note that in alphavirus E1 the conformation of the ij and cd loops provides space for a bulkier GPL head than for the flavi- and phleboviruses. (B and C) Sequence alignment of the bdc $\beta$-sheet region of domain II in flaviviruses (B) and alphaviruses (C). The secondary elements are indicated at the top. A magenta background marks strictly conserved residues across each genus. The cysteines in the conserved disulfide bond and the positively charged residues at the beginning of the $b c$ loop are highlighted by green and blue backgrounds, respectively. Note that whenever the first residue in the bc loop is not lysine (as in MIDDV and WNV), there is a lysine instead as the penultimate residue of strand $b$, which according to our modeling would be in a position to make a similar contact with the phosphate group of the GPL head. See the supplementary materials for the full names of the viruses and accession codes. Single-letter abbreviations for the amino acid residues are as follows: A, Ala; C, Cys; D, Asp; E, Glu; F, Phe; G, Gly; H, His; I, Ile; K, Lys; L, Leu; M, Met; N, Asn; P, Pro; Q, Gln; R, Arg; S, Ser; T, Thr; V, Val; W, Trp; and Y, Tyr. 
group, whereas the interaction of the DOPCtrimethylamine group with the D961 side chains was more dynamic. Indeed, the methyl groups partially shield the positive charge of the central amine, resulting in frequent on-off interactions (fig. S7B), in line with the observed stronger binding to PE-containing membranes (Fig. 2C, left panel). Finally, to rationalize the experimentally determined lack of binding of the W821H mutant and the W821A/F826A double mutant (Fig. 2 and fig. S4D), we computed the change in membrane binding affinity $\Delta \Delta G$ caused by the mutations (fig. S6B). We obtained $\Delta \Delta G$ values of 45.9 and $60.2 \mathrm{~kJ} \mathrm{~mol}^{-1}$ for the single- and double-mutant trimers, respectively, confirming the crucial role of these fusion loop aromatic side chains for binding. For the class I fusion protein hemagglutinin (HA) of influenza virus, the membrane binding energy of the fusion peptide was measured to be roughly $32 \mathrm{~kJ} \mathrm{~mol}^{-1}$ (34), which implies $96 \mathrm{~kJ} \mathrm{~mol}^{-1}$ per HA trimer. The HA fusion peptide inserts nine nonpolar side chains into the membrane (15), a considerably more hydrophobic surface, with a total of 27 hydrophobic residues per trimer. Although the obtained $\Delta \Delta G$ figures are relative (wild type versus mutant) and not absolute binding affinities-and thus cannot be directly compared with the HA case-they still reveal that the effect of inserting only two aromatic side chains per RVFV Gc protomer involves a considerable difference in binding energy, which appears to be within a factor of 2 , at most, from the absolute value measured for the HA trimer.

Inspection of the structures of the flavivirus $\mathrm{E}$ and alphavirus $\mathrm{E} 1$ fusion proteins revealed the presence of a groove at the same location of the GPL pocket of RVFV Gc (Fig. 4A, middle and right panels, respectively). The disulfide bond that interacts with the glycerol moiety is conserved, and a positively charged arginine or lysine side chain at the end of $\beta$-strand $b$ is also in position to interact with the phosphate group. Moreover, the $i j$ loop is also poised to provide the relevant contact that would specify the particular GPL being recognized. In line with the reported dengue virus requirement for anionic GPLs such as phosphatidyl serine in the target membrane for fusion (35), the $i j$ loop in protein E projects a lysine side chain into the pocket (Fig. 4A, middle panel). To test the GPL requirement for binding, we ran liposome coflotation experiments (fig. S8) using the ectodomain of flavivirus E protein (from Zika virus; fig. S8A) and the alphavirus E1 (from chikungunya virus; fig. S8B), confirming that in the absence of GPLs, these proteins display no detectable interaction with membranes, even in the presence of cholesterol to provide space between the bulky lipid heads, similar to the SM and cholesterol results in the case of RVFV Gc (fig. S5B). In contrast, the control herpesvirus class III fusion protein $\mathrm{gB}$ interacted with liposomes made of SM as long as cholesterol was present, indicating that it does not rely on the recognition of GPLs as class II fusion proteins do (fig. S8C).

Taken together, our data confirm that the arbovirus class II fusion proteins do not restructure their fusion loop upon insertion into the outer leaflet of target membranes but instead have a built-in motif to accommodate GPL head groups and induce a reorganization of the membrane by concentrating cholesterol at the site of insertion. It is likely that cholesterol is not the only small lipid in biological membranes that can play this space-filling role, as flaviviruses still fuse with cholesteroldepleted cells (26). Although our results were obtained by performing simulations with a membrane of very simple composition, reflecting the in vitro experimental conditions used in our liposome-binding experiments, the identified insertion mechanism is likely to be valid for insertion into any membrane.

Our results demonstrate that class II fusion proteins have a different mechanism for insertion into the outer leaflet of the target membrane, compared with class I proteins, and rationalize the requirement of a much less hydrophobic segment to achieve stable insertion. Our findings further highlight the impact of comparative studies on fusion proteins from apparently unrelated viruses to extract mechanistic understanding about their function, as our results point to a possible molecular mechanism explaining the impact of the A226V mutation in chikungunya virus, at the tip of the $i j$ loop (Fig. 4A, right panel). Different mosquito species may indeed display different lipid compositions in the endosomal compartment of the relevant cells for infection, and subtle changes may affect successful virus transmission. It is possible that as PC and PE are very common GPLs in eukaryotic membranes, their recognition by RVFV Gc may be related to the very broad mosquito vector usage of this virus. Our understanding of the insertion mechanism now opens the possibility of targeting the GPL-binding site to develop potentially broad antiviral treatments against phleboviruses, flaviviruses, and alphaviruses, which comprise the agents responsible for the most devastating arboviral diseases currently circulating.

\section{REFERENCES AND NOTES}

1. R. Daubney, J. R. Hudson, P. C. Garnham, J. Pathol. 34 545-579 (1931)

2. R. Swanepoel, J. A. W. Coetzer, in Infectious Diseases of Livestock, J. Coetzer, R. Tustin, Eds. (Oxford Univ. Press, 2004), vol. 1, chap. 95, pp. 1037-1070

3. K. J. Linthicum, S. C. Britch, A. Anyamba, Annu. Rev. Entomol. 61, 395-415 (2016)

4. H. H. Balkhy, Z. A. Memish, Int. J. Antimicrob. Agents 21, 153-157 (2003).

5. V. Chevalier, M. Pépin, L. Plée, R. Lancelot, Euro Surveill. 15 , 19506 (2010)

6. A. N. Freiberg, M. B. Sherman, M. C. Morais, M. R. Holbrook, S. J. Watowich, J. Virol. 82, 10341-10348 (2008)

7. J. T. Huiskonen, A. K. Overby, F. Weber, K. Grünewald, J. Virol. 83, 3762-3769 (2009)

8. M. B. Sherman, A. N. Freiberg, M. R. Holbrook, S. J. Watowich, Virology 387, 11-15 (2009)

9. P. Y. Lozach et al., Cell Host Microbe 10, 75-88 (2011).
10. M. Dessau, Y. Modis, Proc. Natl. Acad. Sci. U.S.A. 110 , 1696-1701 (2013)

11. F. A. Rey, F. X. Heinz, C. Mandl, C. Kunz, S. C. Harrison, Nature 375, 291-298 (1995)

12. J. Lescar et al., Cell 105, 137-148 (2001).

13. S. Igonet, F. A. Rey, Cell 151, 1634-1634.e1 (2012).

14. S. C. Harrison, Virology 479-480, 498-507 (2015)

15. J. L. Lorieau, J. M. Louis, A. Bax, Proc. Natl. Acad. Sci. U.S.A. 107, 11341-11346 (2010)

16. W. Qiang, M. L. Bodner, D. P. Weliky, J. Am. Chem. Soc. 130 , 5459-5471 (2008)

17. B. Apellániz, N. Huarte, E. Largo, J. L. Nieva, Chem. Phys. Lipids 181, 40-55 (2014).

18. S. Bressanelli et al., EMBO J. 23, 728-738 (2004).

19. D. L. Gibbons et al., Nature 427, 320-325 (2004)

20. S. Halldorsson et al., Proc. Natl. Acad. Sci. U.S.A. 113 7154-7159 (2016)

21. Y. Modis, S. Ogata, D. Clements, S. C. Harrison, Nature 427 313-319 (2004).

22. S. Roche, F. A. Rey, Y. Gaudin, S. Bressanelli, Science 315 , 843-848 (2007).

23. D. Bitto, S. Halldorsson, A. Caputo, J. T. Huiskonen, J. Biol. Chem. 291, 6412-6422 (2016)

24. M. Kielian, C. Chanel-Vos, M. Liao, Viruses 2, 796-825 (2010).

25. A. Wilder-Smith et al., Lancet Infect. Dis. 17, e101-e106 (2017).

26. M. Umashankar et al., J. Virol. 82, 9245-9253 (2008)

27. K. A. Tsetsarkin, D. L. Vanlandingham, C. E. McGee, S. Higgs, PLOS Pathog. 3, e201 (2007).

28. I. Schuffenecker et al., PLOS Med. 3, e263 (2006).

29. Materials and methods are available as supplementary materials.

30. J. Pérez-Vargas et al., Cell 157, 407-419 (2014).

31. S. Murakami, K. Terasaki, S. I. Ramirez, J. C. Morrill, S. Makino, PLOS Negl. Trop. Dis. 8, e2746 (2014)

32. J. E. Voss et al., Nature 468, 709-712 (2010)

33. D. L. Gibbons et al., Cell 114, 573-583 (2003).

34. X. Han, L. K. Tamm, Proc. Natl. Acad. Sci. U.S.A. 97 13097-13102 (2000)

35. E. Zaitseva, S. T. Yang, K. Melikov, S. Pourmal, L. V. Chernomordik, PLOS Pathog. 6, e1001131 (2010).

\section{ACKNOWLEDGMENTS}

We thank A. Haouz and the staff at the crystallogenesis facility of Institut Pasteur for help with the robotized system; M. Flamand and C. Tamietti from Institut Pasteur for discussion; all other members of the Institut Pasteur "Unité de Virologie Structurale"; M. C. Vaney, B. Saunier, P. Sylvestre, and the Risk Prevention Unit of the Institut Pasteur for their biosafety and biosecurity expertise; W. Shepard and P. Legrand from Synchrotron Soleil for help during data collection and for beneficial discussions; the staff at beamline ID23-2 at the European Synchrotron Radiation Facility (Grenoble, France); and the staff at beamline PX1 at the Swiss Light Source synchrotron (Villigen, Switzerland). F.A.R. received funding from "Integrative Biology of Emerging Infectious Diseases" Labex (Laboratoire d'Excellence) grant ANR-10-LABX-62-IBEID (French government's "Investissements d'Avenir" program) and P.G.-C. and F.A.R. were funded by Labex IBEID (grant ANR-10-LABX-62-IBEID 4E AAP). K.A. and J.S.H. were supported by the Deutsche Forschungsgemeinschaft (grant HU 1971/1-1). K.A. received a stipend from the International Max Planck Research School for Physics of Biological and Complex Systems. We acknowledge the Gesellschaft für Wissenschaftliche Datenverarbeitung (GWDG) and Norddeutscher Verbund für Hoch- und Höchstleistungsrechnen (HLRN) centers for computing time. The coordinate files of the structures described in this manuscript have been submitted to the Protein Data Bank with IDs 6EGT and 6EGU.

\section{SUPPLEMENTARY MATERIALS}

www.sciencemag.org/content/358/6363/663/suppl/DC1 Materials and Methods

Figs. S1 to S8

Tables S1 to S3

References (36-55)

Movies S1 to S5

24 October 2016; resubmitted 20 July 2017

Accepted 25 September 2017

10.1126/science.aal2712 


\section{Science}

\section{A glycerophospholipid-specific pocket in the RVFV class II fusion protein drives target membrane insertion}

P. Guardado-Calvo, K. Atkovska, S. A. Jeffers, N. Grau, M. Backovic, J. Pérez-Vargas, S. M. de Boer, M. A. Tortorici, G. Pehau-Arnaudet, J. Lepault, P. England, P. J. Rottier, B. J. Bosch, J. S. Hub and F. A. Rey

Science 358 (6363), 663-667.

DOI: $10.1126 /$ science.aal2712

\section{Unmasking class II membrane fusion}

Rift Valley fever virus (RVFV) is transmitted by mosquitos and enters cells through receptormediated endocytosis. The infection process requires class II membrane fusion proteins, which insert a hydrophobic fusion loop into cell membranes and then refold. Guardado-Calvo et al. report the high-resolution crystal structure of RVFV class II fusion protein Gc in its postfusion form complexed with phosphatidylcholine. They find that Gc does not restructure its fusion loop after insertion. Rather, it uses an integrated system that accommodates glycerophospholipid head groups and then initiates membrane reorganization by concentrating cholesterol at the insertion site. Comparison with class II fusion proteins from other virus families suggests a common mechanism, which may provide a target for future antiviral therapies.

Science, this issue p. 663

ARTICLE TOOLS

SUPPLEMENTARY

MATERIALS

REFERENCES

PERMISSIONS http://science.sciencemag.org/content/358/6363/663

http://science.sciencemag.org/content/suppl/2017/11/02/358.6363.663.DC1

This article cites 52 articles, 11 of which you can access for free http://science.sciencemag.org/content/358/6363/663\#BIBL

http://www.sciencemag.org/help/reprints-and-permissions

Use of this article is subject to the Terms of Service

Science (print ISSN 0036-8075; online ISSN 1095-9203) is published by the American Association for the Advancement of Science, 1200 New York Avenue NW, Washington, DC 20005. 2017 (C) The Authors, some rights reserved; exclusive licensee American Association for the Advancement of Science. No claim to original U.S. Government Works. The title Science is a registered trademark of AAAS. 